\title{
Variable Speed Limit Control Method of Freeway Mainline in Intelligent Connected Environment
}

\author{
Xingju Wang $\mathbb{D}^{1,2}$ Rongqun Zhang $\mathbb{D}^{2},{ }^{2}$ Yang Gou $\mathbb{D}^{\circ},{ }^{3}$ Jiayu Liu $\mathbb{D}^{2},{ }^{2}$ Lin Zhao, ${ }^{2}$ \\ and Yanting $\mathrm{Li} \mathbb{B}^{2}$ \\ ${ }^{1}$ State Key Laboratory of Mechanical Behavior and System Safety of Traffic Engineering Structures, \\ Shijiazhuang Tiedao University, Shijiazhuang 050043, China \\ ${ }^{2}$ Shijiazhuang Tiedao University, Shijiazhuang 050043, China \\ ${ }^{3}$ China Railway Design Corporation, Tianjin 300000, China \\ Correspondence should be addressed to Xingju Wang; wangxingju@stdu.edu.cn
}

Received 16 July 2020; Revised 26 June 2021; Accepted 14 July 2021; Published 23 July 2021

Academic Editor: Giuseppe Musolino

Copyright $@ 2021$ Xingju Wang et al. This is an open access article distributed under the Creative Commons Attribution License, which permits unrestricted use, distribution, and reproduction in any medium, provided the original work is properly cited.

Freeway is an important component of transportation system. Bottleneck areas on freeway reduce driving safety and traffic efficiency. The development of intelligent connected technology provides a new idea for traffic management. In order to alleviate traffic congestion on the freeway bottleneck area, this paper proposes a variable speed limit (VSL) control method in intelligent connected environment. In this paper, the METANET model is improved by combining intelligent connected environment and VSL control theory. The total traffic capacity (TTC), total travel time (TTT), and total speed difference (TSD) are used to build multiobjective function. The microsimulation at SUMO by using the data from PeMS is employed as a case study to validate the proposed model. The results show that the VSL online control method in intelligent connected environment has better control effect. And the improvement is more obvious with increasing penetration rate of intelligent connected vehicle (ICV).

\section{Introduction}

Due to the rapid growth of freeway transport volume and the influence of holiday travel peak, large-scale congestion of freeway has become an increasingly serious problem. Congestion often occurs where the road segment is temporarily closed for construction. Bottleneck areas, such as the intersection of the main line and the ramp, are also likely to cause congestions. Traditionally static speed limit control technology of freeway cannot meet the existing complex traffic environment.

Many studies have proposed different approaches and models to reduce traffic congestion and delay [1]. Variable speed limit control is a method of traffic management commonly used in freeway systems [2]. Grumert and Tapani [3] summarized important characteristics that affect performance of VSL system, indicating that different characteristics and control algorithms have different impact on traffic performance. Cao et al. [4] analyzed the congestion distribution in various situations under VSL control and non-VSL control. The key of the VSL control is the analysis of the characteristics of traffic state. Therefore, whether the traffic flow model is reasonable directly affects the application effect of the VSL control strategy. Festa et al. [5] analyzed two traffic models in noncongested traffic conditions and discussed models potentialities and limits in largescale applications. Yang et al. [6] proposed an active VSL control method by minimizing two control objectives, travel time minimization and velocity variance minimization, and found that the coincidence rate of drivers was an important factor for the consistency between reality and VSL system. Hegyi et al. [7] presented a model predictive control (MPC) approach to optimally coordinate variable speed limit for freeway traffic. Li and Ranjitkar $[8,9]$ designed a VSL control algorithm based on fuzzy logic in 2015 and a method based on particle swarm optimization in 2019. Yang et al. [10] adopted the Kalman filter VSL control system enhancement module based on the macroscopic traffic flow model to solve 
the displacement capacity of the upstream and downstream of the freeway. Kotsialos et al. [11] used the second-order traffic flow METANET model as a core model, which had good expression characteristics for VSL control. These experiment results showed that advanced models can effectively improve the traffic capacity of road congested sections, including SCTM-METANET [12], proactive VSL based on Dyna TAM-VSL [13], a variety of vehicles model [14], and predictive control based on the cell transmission model [15].

ICV is equipped with advanced sensors, controllers, and modern communication technology, which not only provide more real-time information to infrastructure but also can be controlled and optimized [16]. Traffic flow on the road is generally mixed with ICVs and manual vehicles for a long time to come [17]. Optimal traffic control strategies contribute to reducing congestion, travel time, and fuel consumption [18]. The introduction of intelligent connected technology can optimize traffic flow, reduce total travel time on the road [19], optimize control distance and vehicle performance $[20,21]$, and decrease the vehicle delay and fuel consumption [22]. The construction method of intelligent transportation system based on ICV and big data technology had also been discussed [23]. Cellular automaton model containing the collaborative components of the network and autonomous vehicles (CAVs) [24] and MVDE model considering the interaction between front and rear vehicles [25] both confirmed the important role of intelligent connected technology to improve transport service level. Furthermore, Grumert and Tapani [26] confirmed VSL system can manage to decrease the difference in speeds between individual vehicles by proposed evaluation method. Guo et al. [27] proposed an effective real-time traffic information sharing mechanism which is VANET-assisted. Song et al. [28] proposed a dynamic vehicle path guidance model based on global adaptive optimization scheduling under the Internet of vehicles. Erdağ 1 et al. [29] created two hypothetical test networks with different levels of complexity, in which total time spent and total emission were considered to find an optimum penetration rate of CACC in urban road. Yao et al. [30] established a method for the stability of mixed traffic flow and obtained the fundamental diagram model under different penetration rates of CAVs.

Many studies have applied VSL control to freeway management control system. Most of these works are based on historical traffic data, which cannot reflect the timevarying of traffic flow parameters. The control objective is single, and the method to solve the objective function is a trial algorithm that is difficult to be applied to complex control system. Few studies discuss the adaptive improvement of freeway control methods according to the characteristics of intelligent connected technology. In this paper, the METANET model is optimized and improved by considering characteristics of intelligent connected environment. The VSL control objective function is proposed by maximizing TTC and minimizing TTT and TSD. Finally, the microsimulation is used to verify the effectiveness of the proposed methodology.

\section{Methodology}

2.1. Enhanced METANET Model. As shown in Figure 1, the typical freeway section is divided into $N$ segments with length of $l$. The METANET model calculates the state of traffic flow at the next time interval based on the recurrence formula of the model using the flow, speed, and density of the subsection.

For each segment $i$, the relationship between average traffic volume, average traffic density, and average traffic speed during time interval $k$ can be expressed as

$$
q_{i}(k)=\rho_{i}(k) v_{i}(k) \lambda_{i},
$$

where $q_{i}(k)$ is average volume of vehicles on segment $i$ during time interval $k ; \rho_{i}(k)$ is average density on segment $i$ during time interval $k ; v_{i}(k)$ is average speed on segment $i$ during time interval $k ; \lambda_{i}$ is the number of lanes.

The density can be determined by on-ramp flow and offramp flow as

$$
\rho_{i}(k+1)=\rho_{i}(k)+\frac{T}{\lambda_{i} l_{i}}\left(q_{i-1}(k)-q_{i}(k)+r_{i}(k)-s_{i}(k)\right),
$$

where $T$ is discrete time step used in the METANET model; $l_{i}$ is the length of segment $i ; r_{i}(k)$ is the on-ramp traffic volume on the segment $i$ during time interval $k ; s_{i}(k)$ is the off-ramp traffic volume on the segment $i$ during time interval $k$.

In METANET model, the convection of the speed dynamic function is more consistent with the actual situation in the free flow state. However, under the condition of congestion, this is relatively deviated from the actual operating state. This paper enhanced the third term to alleviate the impact of upstream speed value on downstream traffic flow. Improved speed dynamics formula [31] is as follows:

$$
\begin{aligned}
v_{i}(k+1)= & v_{i}(k)+\frac{T}{\tau}\left[V\left[\rho_{i}(k)\right]-v_{i}(k)\right]+\frac{T}{l_{i}} v_{i}(k)\left[\sqrt{\frac{v_{i-1}^{2}(k)+v_{i}^{2}(k)}{2}}-v_{i}(k)\right] \\
& -\frac{1}{\tau}\left[\frac{T v}{L_{i}} \frac{\rho_{i+1}(k)-\rho_{i}(k)}{\rho_{i}(k)+\kappa}\right]
\end{aligned}
$$




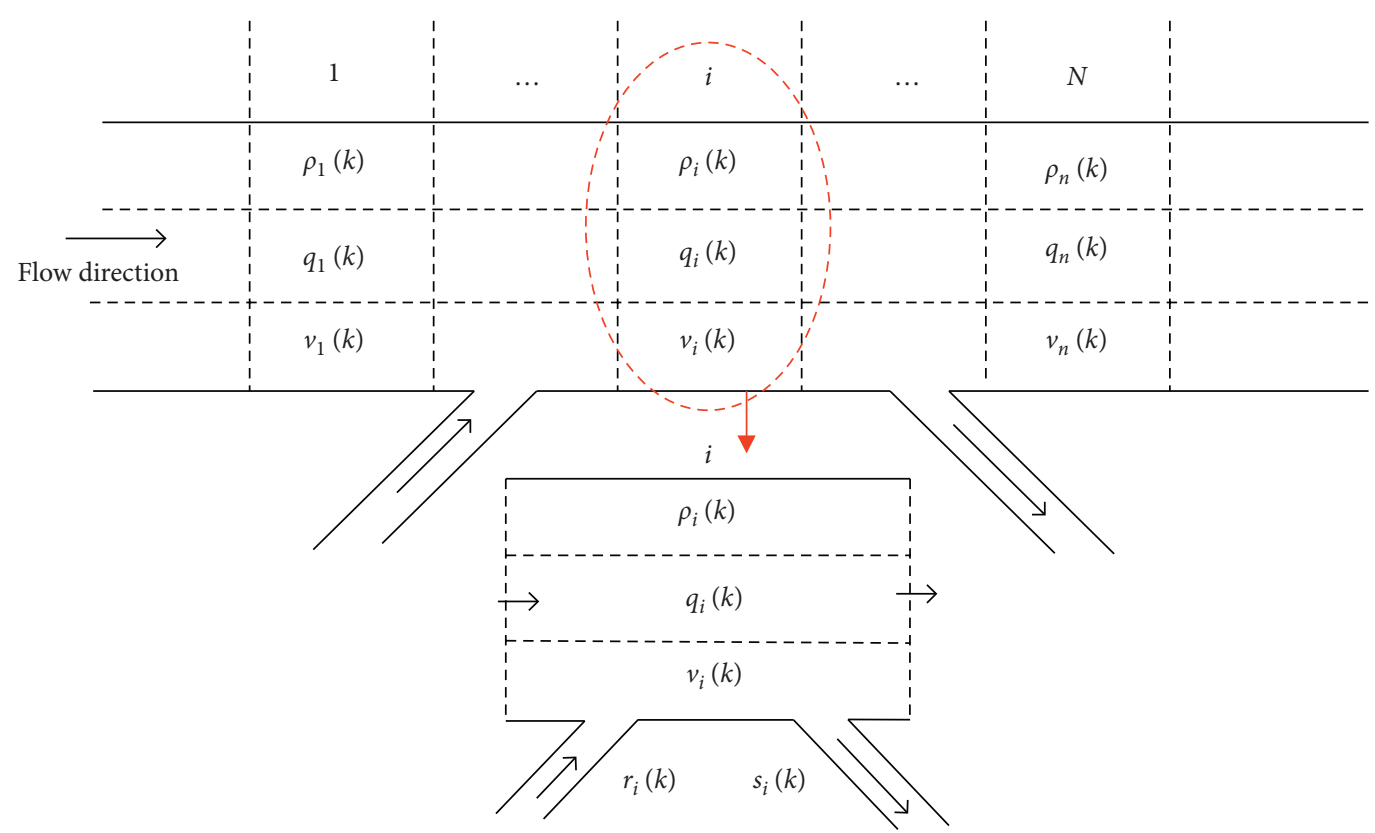

Figure 1: Typical freeway segment diagram.

where $\tau, v$, and $\kappa$ are global parameters of METANET model, determined jointly by characteristics such as road conditions, driver behavior, and vehicle performance.

For most traffic flow models, under the condition of no speed limit control, the driver's desired speed is free flow speed. However, in the VSL control environment, the driver's desired speed is not free flow speed, but the modified speed limit of the road section; that is, each road section aims to control the driver's desired speed below the reasonable speed limit value. Improved driver's desired speed [32] can be expressed as follows:

$$
V\left[\rho_{i}(k)\right]=u_{i}(k) \exp \left[-\frac{1}{o_{i}}\left(\frac{\rho_{i}(k)}{\rho_{c}}\right)^{o_{i}}\right],
$$

where $o_{i}$ is set to 2 in this paper; $u_{i}(k)$ is the speed limit value on segment $i$ during time interval $k$.

Compared with manual vehicles, ICV has a higher compliance rate. The maximum speed of a vehicle can be adjusted according to vehicles with higher compliance rate [33]. Therefore, in the intelligent connected environment, the control center improves the speed limit value of each control section by collecting real-time data from the road traffic state monitoring device and the ICV. This can be expressed as follows [14]:

$$
\begin{aligned}
u_{i}^{\mathrm{acc}}(k) & =\left(1+\alpha_{i}(k)\right) \times u_{i}(k), \\
\alpha_{i}(k) & =\frac{v_{i}^{\mathrm{acc}}(k-1)-u_{i}^{\mathrm{acc}}(k-1)}{u_{i}^{\mathrm{acc}}(k-1)},
\end{aligned}
$$

where $u_{i}^{\text {acc }}(k)$ is the modified speed limit in the intelligent connected environment on segment $i$ during time interval $k$; $\alpha_{i}(k)$ is the relation coefficient between the ICV speed and the speed limit on segment $i$ during time interval $k$; $v_{i}^{\text {acc }}(k-$ 1 ) is average speed of ICV on segment $i$ during time interval $k-1 ; u_{i}^{\text {acc }}(k-1)$ is the speed limit on segment $i$ during time interval $k-1$.

2.2. Control Objective Function. On the premise of ensuring the traffic capacity of the road, the control objective function is established by combining TTC, TTT, and TSD to improve the traffic efficiency of the road and the driving comfort. The weight relationship among the three terms is balanced to realize the coordinated control of VSL.

Increasing traffic volume on the road segment can improve the efficiency of the road traffic. The TTC as one of control objectives during the predicted time length $N p$ can be expressed as

$$
\mathrm{TTC}=T \sum_{k=1}^{N_{p}} \sum_{i=1}^{N} \lambda_{i} l_{i} \rho_{i}(k) v_{i}(k) .
$$

Reducing the TTT can effectively improve the efficiency of road traffic. The TTT as one of control objectives can be expressed as

$$
\operatorname{TTT}=T \sum_{k=1}^{N_{p}} \sum_{i=1}^{N} \lambda_{i} l_{i} \rho_{i}(k) .
$$

When the difference of driver's speed changes is large, the vehicle will accelerate and decelerate to a large extent. It is easy to cause traffic collisions and to reduce stability of traffic flow. The TSD selected as the control target can be expressed as

$$
\operatorname{TSD}=\sum_{k=1}^{N_{p}} \sum_{i=1}^{N}\left(u_{i}^{a c c}(k)-v_{i}(k)\right)^{2} .
$$

In this paper, control objective function includes TTC, TTT, and TSD. In the optimal state, TTC is the maximum 
while TTT and TSD are the minimum. In this case, the speed limit value is the optimal solution of our optimization model. The multiobjective function normalizes TTC, TTT, and TSD to obtain $S_{1}, S_{2}$, and $S_{3}$ and combines with the corresponding weight. Then the optimal speed limit value is obtained by minimization of multiobjective function. It can be expressed as

$$
\begin{aligned}
& S_{1}=\frac{\mathrm{TTC}-\mathrm{TTC}_{\min }}{\mathrm{TTC}_{\max }-\mathrm{TTC}_{\min }} \\
& S_{2}=\frac{\mathrm{TTT}-\mathrm{TTT}_{\min }}{\mathrm{TTT}_{\max }-\mathrm{TTT}_{\min }}, \\
& S_{3}=\frac{\mathrm{TSD}-\mathrm{TSD}_{\min }}{\mathrm{TSD}_{\max }-\mathrm{TSD}_{\min }} \\
& \operatorname{Min}\left\{-w_{1} \frac{\mathrm{TTC}_{1}-\mathrm{TTC}_{\min }}{\mathrm{TTC}_{\max }-\mathrm{TTC}_{\min }}+w_{2} \frac{\mathrm{TTT}_{2}-\mathrm{TTT}_{\min }}{\mathrm{TTT}_{\max }-\mathrm{TTT}_{\min }}+w_{3} \frac{\mathrm{TSD}-\mathrm{TSD}_{\min }}{\mathrm{TSD}_{\max }-\mathrm{TSD}_{\min }}\right\},
\end{aligned}
$$

where $w_{1}, w_{2}$, and $w_{3}$ are weight parameter of TTC, TTT, and $\mathrm{TSD}$, respectively; $\mathrm{TTC}_{\max }$ and $\mathrm{TTC}_{\min }$ are the maximum and minimum of TTC, respectively; $\mathrm{TTT}_{\max }$ and TTT $_{\text {min }}$ are the maximum and minimum of TTT, respectively; $\mathrm{TSD}_{\max }$ and $\mathrm{TSD}_{\min }$ are the maximum and minimum of TSD, respectively.

The weight was determined by the 1-9 scale method in the analytic hierarchy process to calibrate the importance of the control target. The quantitative rules of the 1-9 scale method are shown in Table 1.

By analyzing the importance of these three functions, the judgment matrix of the objective function is obtained, as shown in Table 2.

The weight coefficient of each objective function is calculated according to the square root method, which can be expressed as

$$
w_{i}=\left(\prod_{j=1}^{n} a_{i j}\right)^{1 / n}
$$

where $a_{i j}$ is the element value of the $i$ th row and $j$ th column in the target judgment matrix.

Normalization according to the formula can be expressed as

$$
w=\frac{w_{i}}{\sum_{i}^{n} w_{i}}
$$

The weight calculation results are shown in Table 3.

The consistency test is carried out on the results in Table 3 and the verification result $\mathrm{CR}=0.0474<0.1$, which is in line with inspection standards.

The speed limit value of the freeway should be controlled within a reasonable range to ensure the comfort and safety of driving behavior while improving the traffic efficiency of the freeway. Our multiobjective function is given by the following formula:

$$
\operatorname{Min}\left\{-0.6491 \frac{\mathrm{TTC}-\mathrm{TTC}_{\min }}{\mathrm{TTC}_{\max }-\mathrm{TTC}_{\min }}+0.2790 \frac{\mathrm{TTT}-\mathrm{TTT}_{\min }}{\mathrm{TTT}_{\max }-\mathrm{TTT}_{\min }}+0.0719 \frac{\mathrm{TSD}-\mathrm{TSD}_{\min }}{\mathrm{TSD}_{\max }-\mathrm{TSD}_{\min }}\right\}
$$

It is subject to

$$
\begin{aligned}
u_{i}^{\mathrm{acc}}(k) & \leq V_{\max }, \\
u_{i}^{\mathrm{acc}}(k) & \geq V_{\min }, \\
\left|u_{i}^{a c c}(k+1)-u_{i}^{\mathrm{acc}}(k)\right| & \leq 20 \frac{\mathrm{km}}{\mathrm{h}},
\end{aligned}
$$


TABLE 1: 1-9 scale quantization.

\begin{tabular}{lc}
$\begin{array}{l}\text { Scale } \\
\text { value }\end{array}$ & Description \\
\hline 1 & Same importance \\
3 & The former is slightly more important than the latter \\
5 & The former is significantly more important than the \\
7 & The former is more important than the latter \\
9 & The former is extremely more important than the \\
$2,4,6,8$ & latter \\
\hline
\end{tabular}

TABLE 2: Objective function judgment matrix.

\begin{tabular}{lccc}
\hline Objective function & $S_{1}$ & $S_{2}$ & $S_{3}$ \\
\hline$S_{1}$ & 1 & 3 & 7 \\
$S_{2}$ & $1 / 3$ & 1 & 5 \\
$S_{3}$ & $1 / 7$ & $1 / 5$ & 1 \\
\hline
\end{tabular}

TABLE 3: Weight calculation results.

\begin{tabular}{lccc}
\hline Objective function & $S_{1}$ & $S_{2}$ & $S_{3}$ \\
\hline Weight & 2.7589 & 1.1856 & 0.3057 \\
Normalized result $(w)$ & 0.6491 & 0.2790 & 0.0719 \\
\hline
\end{tabular}

$$
\begin{aligned}
& \left|u_{i+1}^{\mathrm{acc}}(k)-u_{i}^{\mathrm{acc}}(k)\right| \leq 20 \frac{\mathrm{km}}{\mathrm{h}}, \\
& u_{i}^{\mathrm{acc}}(k) \in\left\{\begin{array}{c}
40 \frac{\mathrm{km}}{\mathrm{h}}, 45 \frac{\mathrm{km}}{\mathrm{h}}, 50 \frac{\mathrm{km}}{\mathrm{h}}, 55 \frac{\mathrm{km}}{\mathrm{h}} \\
\ldots 105 \frac{\mathrm{km}}{\mathrm{h}}, 110 \frac{\mathrm{km}}{\mathrm{h}}, 115 \frac{\mathrm{km}}{\mathrm{h}}, 120 \frac{\mathrm{km}}{\mathrm{h}}
\end{array}\right\}, \\
& \rho_{i}(k+1)=\rho_{i}(k)+\frac{T}{\lambda_{i} l_{i}}\left(q_{i-1}(k)-q_{i}(k)+r_{i}(k)-s_{i}(k)\right), \\
& v_{i}(k+1)=v_{i}(k)+\frac{T}{\tau}\left[V\left[\rho_{i}(k)\right]-v_{i}(k)\right]+\frac{T}{l_{i}} v_{i}(k)\left[\sqrt{\frac{v_{i-1}^{2}(k)+v_{i}^{2}(k)}{2}}-v_{i}(k)\right] \\
& -\frac{1}{\tau}\left[\frac{T v}{L_{i}} \frac{\rho_{i+1}(k)-\rho_{i}(k)}{\rho_{i}(k)+\kappa}\right] \\
& V\left[\rho_{i}(k)\right]=\frac{u_{i}^{\mathrm{acc}}(k)}{\left(1+\left(\left(v_{i}^{\mathrm{acc}}(k-1)-u_{i}^{\mathrm{acc}}(k-1)\right) / u_{i}^{\mathrm{acc}}(k-1)\right)\right)} \exp \left[-\frac{1}{o_{i}}\left(\frac{\rho_{i}(k)}{\rho_{c}}\right)^{o_{i}}\right]
\end{aligned}
$$

Formula (13) takes into account the safety of the open freeway. The speed limit value should be less than the maximum speed limit of the freeway. Formula (14) considers the characteristics of high speed, high efficiency, and high traffic volume of freeway. The speed limit value should be higher than the minimum speed limit so that the freeway can realize its normal road transportation function. Formulas (15) and (16) are to consider the driver's driving comfort and driving safety and to prevent the driver from causing a traffic accident due to excessive acceleration and deceleration in the VSL area. The difference between the speed limits on the controlled road section during the two adjacent time intervals should not exceed the maximum value of driver's speed change. Formula (17) is to consider driver's maneuverability to driving speed, and the speed interval is set to an integer multiple of $5 \mathrm{~km} / \mathrm{h}$. Formulas (18) to (20) are dynamic constraints from enhanced METANET model.

2.3. Control Flow. The traditional method of formulating VSL control strategies are based on historical traffic flow data through technical experience. That is no longer applicable to the rapid development of information technology because of neglecting the time-varying nature of traffic data. As shown in Figure 2, this paper constructs an online control method and formulates control strategies based on the time-varying nature of traffic flow.

The principle of VSL system is as follows: the fundamental traffic flow data from monitors of freeway system is transferred to the internal controller after analysis and processing. Internal controller calculates traffic flow prediction by the enhanced METANET model from time interval $k$ to the predicted time. Then the traffic flow data can be passed to the optimization model. The control objective function is solved to get the speed limit of target section. The optimal solution of the optimization problem is fed back to VSL control system. Due to realtime information interaction and acquisition of intelligent connected environment, the variable speed limit can be dynamically updated so as to continuously optimize and achieve global optimization.

Data collection: collect and update traffic flow data in real time using the detectors set in the freeway and intelligent connected vehicles.

METANET model: predict traffic flow data by the acquired data from freeway system.

Optimization model: achieve optimized control target according to different control objectives, such as reducing 


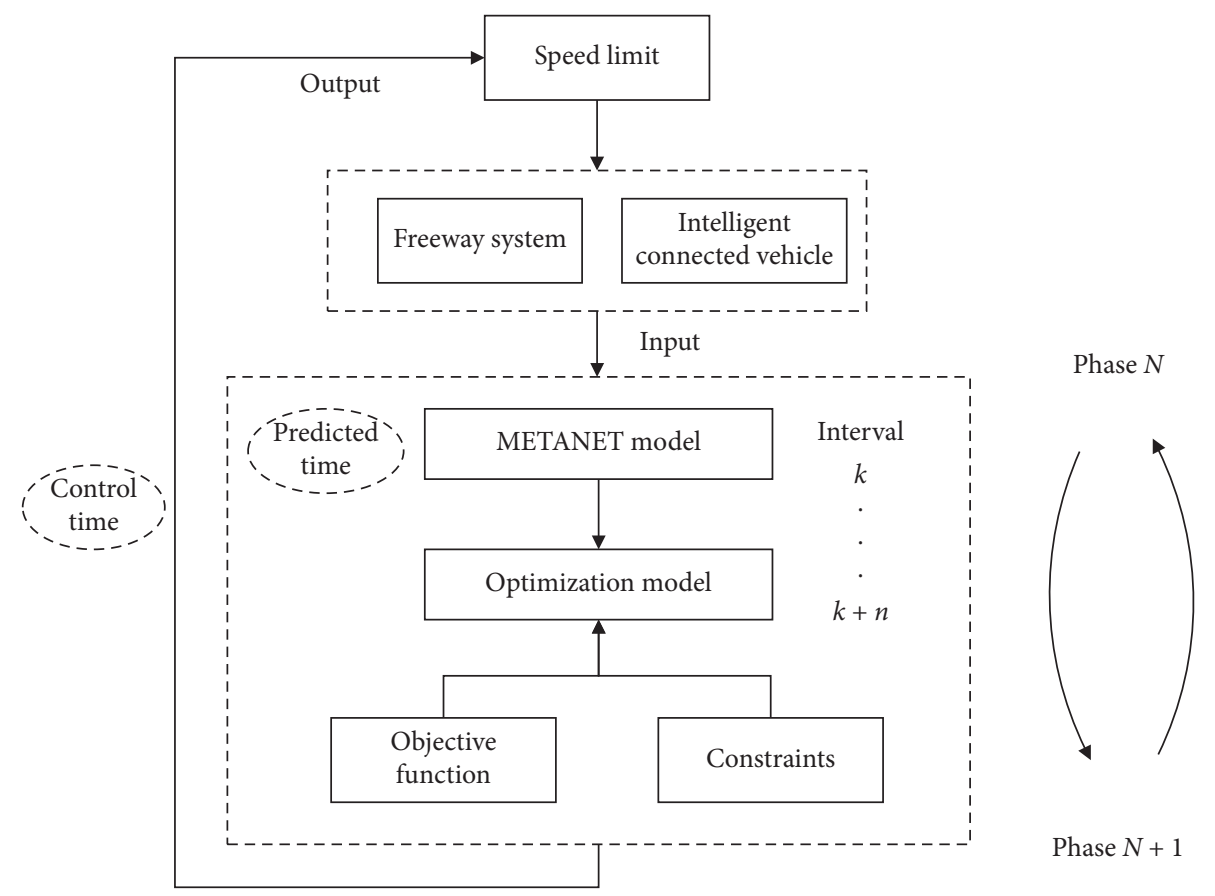

Figure 2: Control flow diagram.

travel time and increasing total traffic flow by the objective function and constraint equations.

Solving tool: solve the control objective function within the predicted time, get the speed limit value of each road segment, and feed back to the freeway to realize the control within the control time.

The control model contains two important parameters, prediction time length $N p$ and control time length $N c$, and the values are generally an integer multiple of discrete time step $T$. The combination of the prediction time length and the control time length will directly affect the control effect $[34,35]$. The length of the prediction time should not be less than the travel time consumed by the vehicle in the control section. The excessively large prediction step will increase the calculation cost and is not conducive to simulation experiment. When the traffic flow is close to congestion, the traffic flow state is extremely unstable. The controller step should be updated in real time to ensure that measures can be taken to the traffic state changes. In this article, the value of $N p$ is set to $5 \mathrm{~min}$ and $N c$ is set to $3 \mathrm{~min}$.

\section{Case Study}

3.1. Simulation Scenario. Simulation of Urban Mobility (SUMO) is an open source, multimodal traffic simulation software that can modify vehicle state, operation mode, and other parameters to a greater extent based on the user's algorithm control requirements. This article selects SUMO as the basic simulation tool. The simulation scenario is as shown in Figure 3, and the road is divided into 7 sections. At the end of the 7 th section, the road is closed due to factors such as construction occupation, and the second section to the 6 th section are set to be in the variable speed limit control area. A traffic detection device is set at the front end of each section of road. The seventh section is the set variable speed limit dissipation area, and the length of the dissipation area is set to $500-700 \mathrm{~m}$. The simulation parameter input is shown in Tables 4 and 5 .

Intelligent connected technology makes vehicles no longer individual driving unit, but an information body that can interact with the driving environment in real time. More information can be obtained between the vehicle and the control center. The operation state of ICV can be adjusted in real time to make these vehicles safer and more reasonable. Therefore, traffic efficiency is effectively improved. The driving behavior of the vehicle is an important factor that affects the performance of the traffic flow. This is also the research basis of the traffic control strategy in the intelligent connected environment.

Treiber [36] proposed an intelligent driver model (IDM) in 2000. The IDM is widely applicable in intelligent connected environment. The ICV has better driving stability and average minimum time headway than manual vehicles. In free flow, the car accelerates to desired speed under the control of the model and keeps running at that speed. Under congested state, when the difference between the front and rear car speeds is not large, small changes in vehicle spacing will not cause the rear car to decelerate. Each parameter of the IDM represents a specific physical meaning. Different driving strategies can be implemented among vehicles by modifying the parameters. Therefore, this paper selects the IDM as the car-following model of intelligent connected vehicle. 


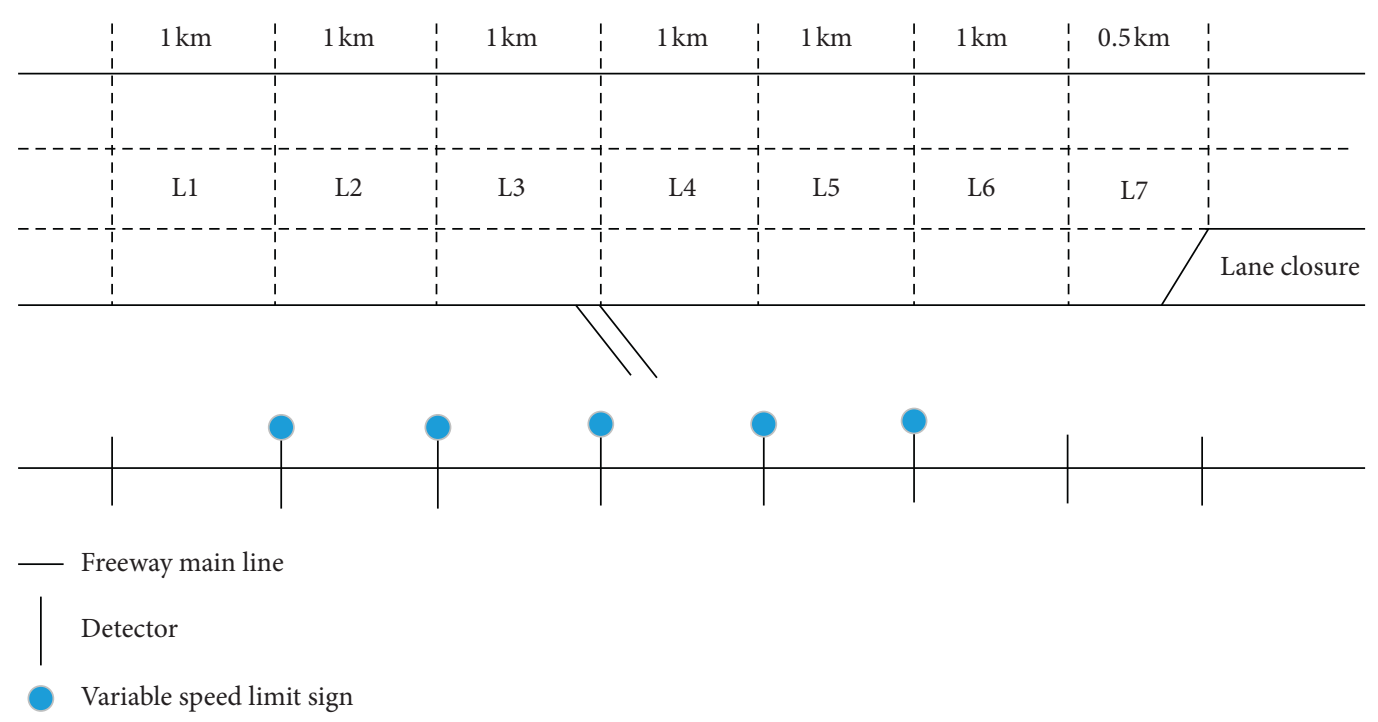

FIgURE 3: Simulation scenario.

TABLE 4: Simulation parameter input.

\begin{tabular}{lcc}
\hline No. & Time interval (s) & Input flow (veh/h) \\
\hline 1 & $0-1000$ & 3000 \\
2 & $1000-5000$ & 4500 \\
3 & $5000-10000$ & 2000 \\
\hline
\end{tabular}

The IDM can be expressed as follows:

$$
\begin{aligned}
& a=a_{0}\left[1-\left(\frac{v_{i}}{v_{0}}\right)^{\delta}+\left(\frac{s_{1}}{s}\right)^{2}\right], \\
& s_{1}=s+T v_{i}+\frac{v_{i} \Delta v_{i}}{2 \sqrt{a_{0} b}},
\end{aligned}
$$

where $a$ is the acceleration of vehicle; $v_{i}$ is the speed of vehicle; $\Delta v_{i}$ is the speed difference between vehicle and the front vehicle; $v_{0}$ is the driver's desired speed in free flow; $a_{0}$ is the maximum acceleration of the vehicle; $b$ is the braking deceleration of the vehicle; $s$ is the minimum safety distance; $T$ is the expected time headway; $\delta$ is the model parameter.

The driving parameters of the IDM of the intelligent connected vehicle in this paper are shown in Table 6 [37].

3.2. Calibration of METANET Model. It can be seen from the METANET model that there are global parameters $\tau, v$, and $\kappa$. They are closely related to the characteristics of road segments, vehicles, and traffic flow. In order to narrow the difference between the METANET model and the actual road conditions, this paper uses the data obtained by the
TABle 5: Simulation scenarios.

\begin{tabular}{lc}
\hline No. & Description \\
\hline Scenario 1 & With no variable speed limit and 100\% manual \\
vehicle
\end{tabular}

TABle 6: The parameter of IDM.

\begin{tabular}{lccccc}
\hline Parameter & $a_{0}\left(\mathrm{~m} \cdot \mathrm{s}^{-2}\right)$ & $b\left(\mathrm{~m} \cdot \mathrm{s}^{-2}\right)$ & $\delta$ & $s(\mathrm{~m})$ & $T(\mathrm{~s})$ \\
\hline ICV & 1 & 2 & 4 & 0 & 1.1 \\
Manual vehicle & 1 & 2 & 4 & 2.5 & 1.6 \\
Truck & 1 & 2 & 4 & 2.5 & 1.6 \\
\hline
\end{tabular}

simulation of the SUMO to calibrate the improved METANET model parameters. To make the parameters more reasonable, the predicted speed and traffic volume of the model are compared with actual data on road sections. The optimization objective function is minimized to reduce the error.

The optimization function can be expressed as 


$$
\min f=\sum_{i=1}^{N} \sum_{k=1}^{K}\left(\left(\frac{v_{i, \text { actual }}(k)-v_{i, \text { predicted }}(k)}{v_{i, \text { actual }}(k)-v_{i, \text { predicted }}(k) / 2}\right)^{2}+\left(\frac{q_{i, \text { actual }}(k)-q_{i, \text { predicted }}(k)}{q_{i, \text { actual }}(k)-q_{i, \text { predicted }}(k) / 2}\right)^{2}\right) .
$$

It is subject to

$$
\begin{aligned}
& X_{\min } \leq X \leq X_{\max }, \\
& X_{\min }=[0.05,10,10], \\
& X_{\max }=[0.2,60,60] .
\end{aligned}
$$

The simulation scenario is the condition with no road closed and the results are shown in Table 7.

\section{Results}

The simulation results are shown in Table 8 . When the intelligent connected vehicle permeability is from 0 to 0.25 , the simulation results show that average travel time, average delay, number of passing vehicles, average $\mathrm{CO} 2$ emissions, and average PMx emissions are all improved by $6.68 \%$, $8.72 \%, 2.37 \%, 2.99 \%$, and $2.39 \%$, respectively. When intelligent connected vehicle permeability is 0.25 and the improved intelligent connected environment VSL control is used, average travel time, average delay, number of passing vehicles, average $\mathrm{CO}_{2}$ emissions, and average $\mathrm{PMx}$ emissions are all improved by $27.43 \%, 46.13 \%, 10.88 \%, 16.55 \%$, and $22.38 \%$, respectively. The control center can obtain the driving information of the ICV at any time to correct the speed limit value. There is an improvement compared with the uncontrolled state. It can be seen that the VSL online control method can more effectively improve the driving state of the road environment and reduce exhaust emissions.

When in scenario 2 situation, the density changes of the fifth and sixth sections are shown in Figure 4. When the simulation time is $4000 \mathrm{~s}$, the congestion spreads from the road closure to the sixth section of the road. At this time, the road section density is higher and the vehicles are in serious congestion state. When the simulation time is $6000 \mathrm{~s}$, the traffic jam phenomenon spreads to the fifth section. When the simulation time is $9000 \mathrm{~s}$, as the queuing gradually dissipates, the congestion state of the fifth road section can be reduced.

When in scenario 4 , that is, with the improved VSL control in the intelligent connected environment, the density changes of the fifth and sixth segments are shown in Figures 5 and 6 . It can be seen from Figures 4 and 5 that the improved VSL control can control the road density by controlling the driving speed of the vehicle on the road section. The density of the road section is significantly reduced. The average driving speed of the vehicle is greatly improved, which greatly eases the phenomenon of traffic congestion and improves the traffic efficiency and driving safety of vehicles.

When in scenario 4 , the flow comparison curve of the sixth section is shown in Figure 7. Before the simulation time $4000 \mathrm{~s}$, there is no congestion phenomenon. The traffic has no change even with the VSL control. When the simulation time is over $4000 \mathrm{~s}$, it can be seen that the improved VSL control system can properly increase the number of vehicles passing and improve the throughput of congested roads.

From the above analysis, it can be seen that the use of improved VSL control in an intelligent connected environment can effectively alleviate traffic congestion in bottleneck areas. It also improves vehicle traffic efficiency and driving stability and effectively reduces $\mathrm{CO}_{2}$ and $\mathrm{PMx}$ emissions, which is beneficial to environmental protection and green transportation.

In the application process of VSL control system, the improvement effect is important, and the operating efficiency of the system also can not be ignored. Long calculating time will lead to a significant reduction on the effectiveness of the system in practical applications. In this system, the calculation part is mainly integrated in the genetic algorithm solution process. The time consumed by the system is mainly from the time occupied by the genetic algorithm solution. In this paper, solution process iterations of the VSL control system are commonly between 60 and 90 times. The convergence speed is relatively fast. It can be seen from Figure 8 that the average running time is around $2 \mathrm{~s}$, which confirms the high efficiency of improved VSL control system.

4.1. Sensitivity Analysis. The relationship between average travel time and the penetration rate of intelligent connected vehicles is shown in Figure 9. As can be seen from Figure 9, the efficiency of road sections is improved and average travel time continues to decrease with continuous increase of the penetration rate of ICV. When penetration rate of ICV is $25 \%, 50 \%$, and $75 \%$, the travel time will be reduced by $6.7 \%$, $14.1 \%$, and $21.2 \%$, respectively, compared with no ICV. Furthermore, the improved VSL control system can have a better improvement effect on average travel time under the conditions of the above penetration rate of ICV. Compared with no VSL control, the proportion of improvement is $27.4 \%, 26.2 \%$, and $26.7 \%$, respectively.

The relationship between average delay and the penetration rate of intelligent connected vehicles is shown in Figure 10. It can be seen that as the penetration rate of ICV continues to increase, the average delay continues to decrease. When penetration rate of ICV is $25 \%, 50 \%$, and $75 \%$, the average delay is reduced by $8.7 \%$, $18.3 \%$, and $27.3 \%$ compared with no ICV. In addition, the improved VSL control system can significantly reduce average delay under the conditions of the above penetration rate of ICV. Compared with no VSL control, the proportion of improvement is $46.1 \%, 50.6 \%$, and $21.2 \%$, respectively.

The relationship between the number of vehicles and the penetration rate of intelligent connected vehicles is shown in Figure 11 . The number of vehicles actually passing through a road segment is one of the most important parameters that 
TABLE 7: Parameter calibration results.

\begin{tabular}{lccccc}
\hline ICV permeability $(\%)$ & $\tau$ & $v$ & $\kappa$ & Free flow speed/(km/h) & Critical density/(veh/(km·lane)) \\
\hline 0 & 0.05 & 13.9196 & 53.9389 & 110 & 13 \\
25 & 0.05 & 13.9196 & 53.9389 & 110 & 15 \\
50 & 0.05 & 13.9196 & 53.9389 & 110 & 17 \\
75 & 0.05 & 13.9196 & 53.9389 & 110 & 19 \\
100 & 0.05 & 13.9196 & 53.9389 & 110 & 22 \\
\hline
\end{tabular}

TABLE 8: Simulation result.

\begin{tabular}{lccccc}
\hline No. & Average travel time (s) & Average delay (s) & $\begin{array}{c}\text { Passing vehicles } \\
(\text { veh })\end{array}$ & $\begin{array}{c}\text { Average } \mathrm{CO}_{2} \text { emissions } \\
(\mathrm{mg})\end{array}$ & Average PMx emissions (mg) \\
\hline Scenario 1 & 812.92 & 621.49 & 5789 & 2067.95 & 44.86 \\
Scenario 2 & 758.65 & 567.27 & 5926 & 2006.04 & 43.79 \\
Scenario 3 & 574.04 & 350.79 & 6550 & 1760.20 & 36.41 \\
Scenario 4 & 550.53 & 305.57 & 6571 & 1674.00 & 33.99 \\
\hline
\end{tabular}

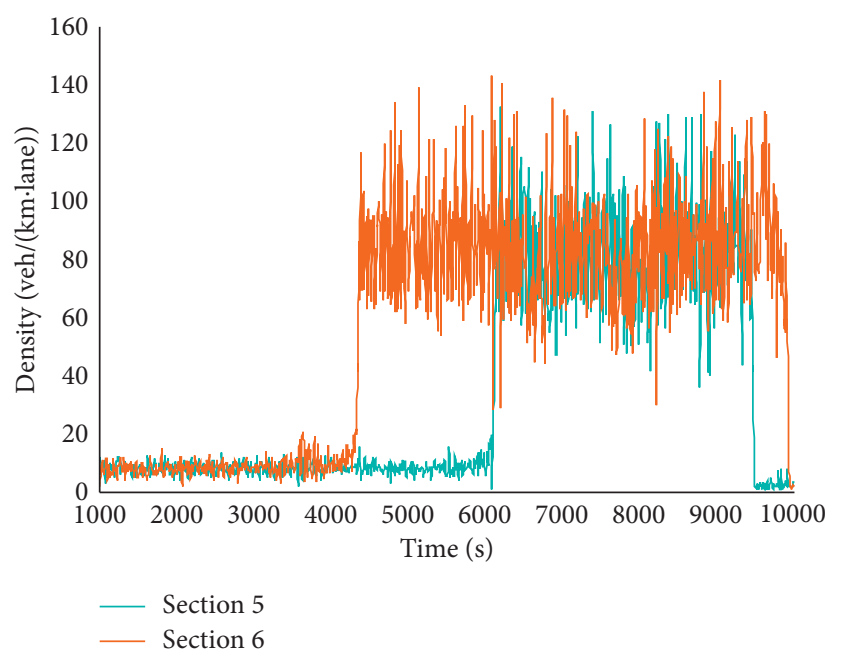

FIgURE 4: Scenario 2 simulation result diagram.

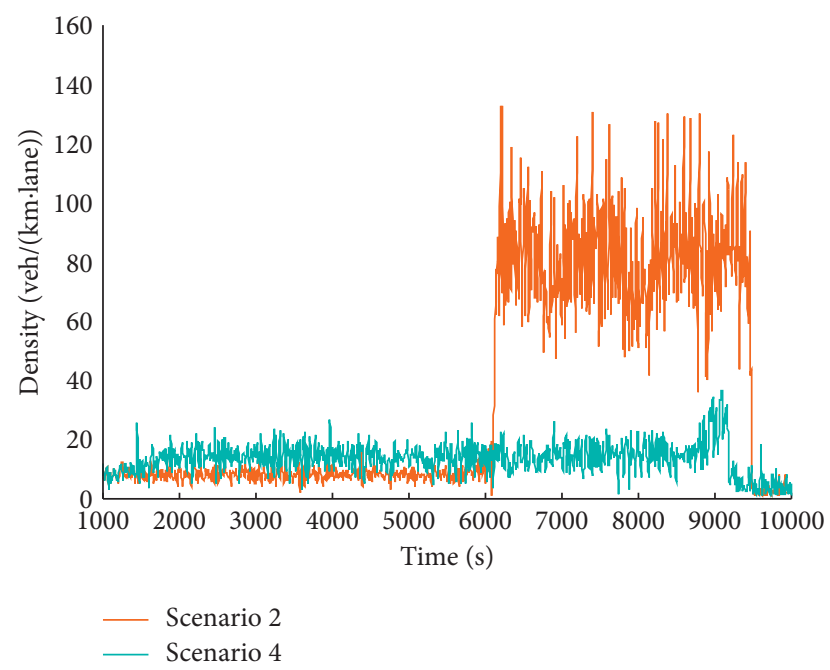

FIgURE 5: The fifth density comparison diagram between scenario 2 and scenario 4 .

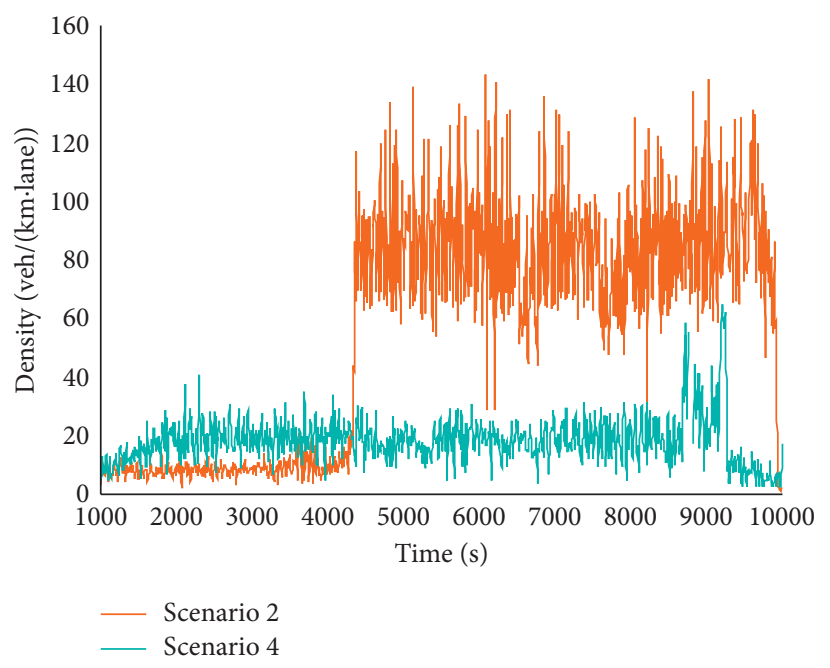

FIGURE 6: The sixth density comparison diagram between scenario 2 and scenario 4.

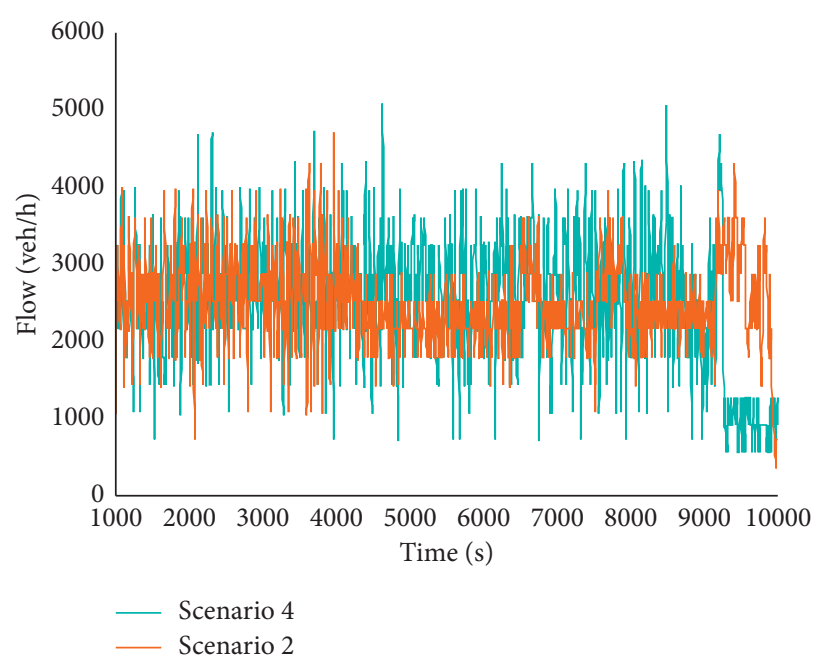

FIgURE 7: The sixth traffic flow comparison diagram between scenario 2 and scenario 4 . 


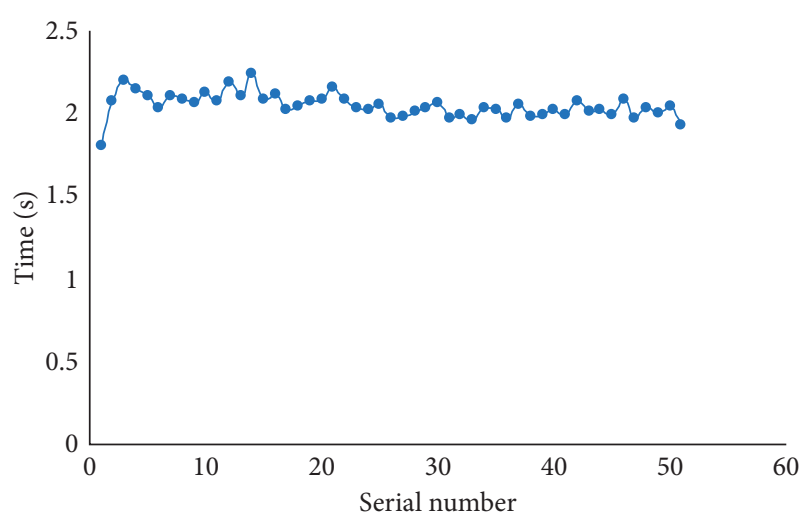

FIgUre 8: The calculating time.

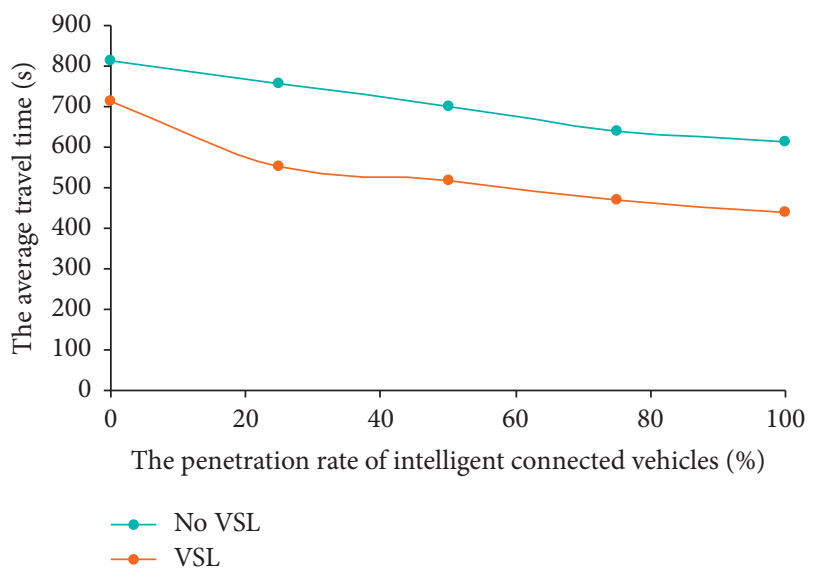

Figure 9: The average travel time result.

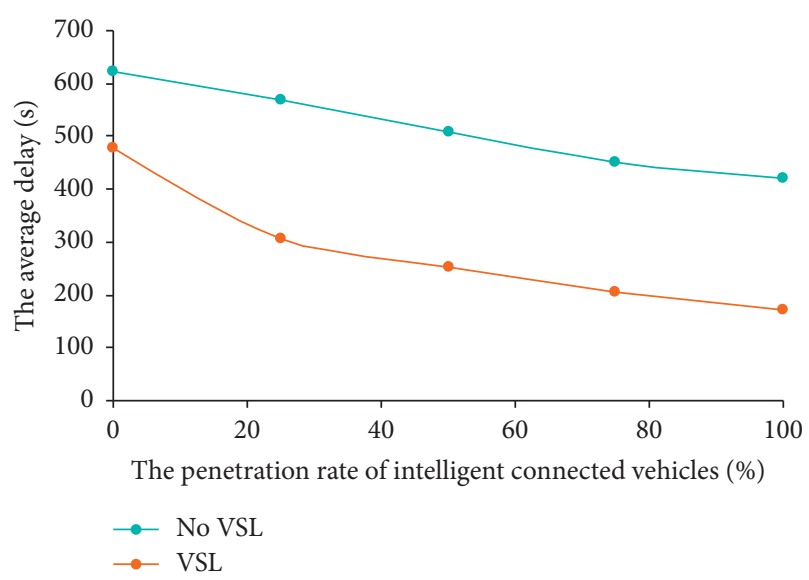

Figure 10: The average delay result.

can directly reflect the actual road capacity. It can be seen from Figure 11 that, with the increasing penetration rate of ICV, the number of actually passing vehicles continues to increase. For example, with ICV permeability by $25 \%, 50 \%$, and $75 \%$, the improvement efficiency is $2.7 \%, 14.7 \%$, and $24.3 \%$ compared with no ICV. In addition, the improved

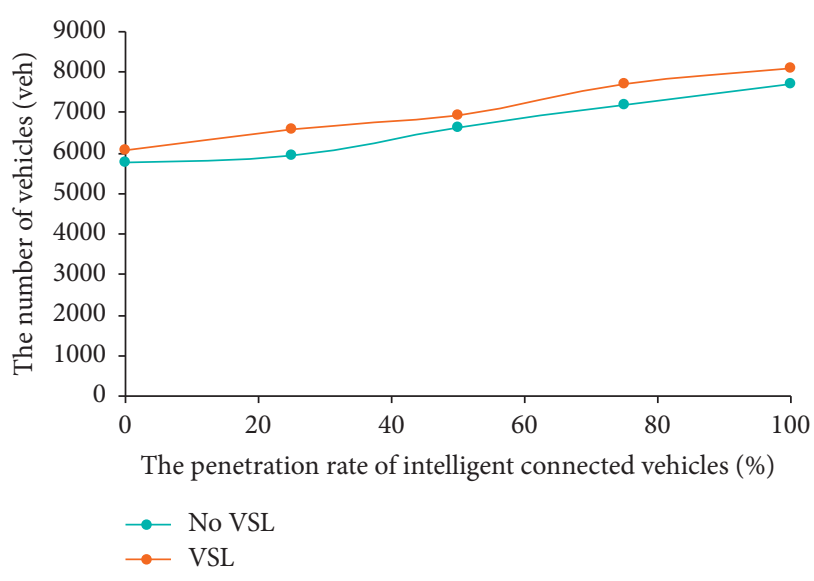

FIGURE 11: The number of vehicles' result.

VSL control system can increase the number of actually passing vehicles in the bottleneck area. Compared with no VSL control, the ratio of improvement is $10.9 \%, 4.6 \%$, and $6.9 \%$, respectively.

\section{Conclusions}

With the innovation and development of intelligent connected technology, ICV will be widely used in real life in the near future. In this paper, considering the characteristics of intelligent connected technology, the VSL online control method combined intelligent connected technology and VSL theory to optimize the VSL control system. The improved VSL control system in intelligent connected environment has a better improvement effect under different ICV penetration rates. The increase of ICV permeability can further alleviate the traffic congestion, which can effectively improve the traffic efficiency and ease the congestion in the bottleneck area.

\section{Data Availability}

The data used to support the findings of this study are available from the corresponding author upon request.

\section{Conflicts of Interest}

The authors declare that there are no conflicts of interest regarding the publication of this paper.

\section{Acknowledgments}

This research was sponsored by the key project supported by the Scientific Research Foundation, Education Department of Hebei Province of China (ZD2021336). This work was further sponsored by the Social Science Development Research Project of Hebei Province of China (No. 20200302079).

\section{References}

[1] B. Alonso, Á. I. Pòrtilla, G. Musolino, C. Rindone, and A. Vitetta, "Network Fundamental Diagram (NFD) and traffic 
signal control: first empirical evidences from the city of Santander," Transportation Research Procedia, vol. 27, pp. 27-34, 2017.

[2] M. Sadat and H. B. Celikoglu, "Simulation-based variable speed limit systems modelling: an overview and a case study on Istanbul freeways," Transportation Research Procedia, vol. 22, pp. 607-614, 2017.

[3] E. F. Grumert and A. Tapani, "Characteristics of variable speed limit systems," European Transport Research Review, vol. 10 , no. 2 , pp. 1-12, 2018.

[4] J. Cao, D. Hu, Y. Luo, T. Z. Qiu, and Z. Ma, "Exploring the impact of a coordinated variable speed limit control on congestion distribution in freeway," Journal of Traffic and Transportation Engineering (English Edition), vol. 2, no. 3, pp. 167-178, 2015.

[5] D. C. Festa, G. Longo, G. Mazzulla et al., "Experimental analysis of different simulation models for motorway traffic flow," in Proceedings of 2001 IEEE Intelligent Transportation Systems, Oakland, CA, USA, August 2001.

[6] X. Yang, Y. C. Lu, G.-L. Chang et al., "Exploratory analysis of an optimal variable speed control system for a recurrently congested freeway bottleneck," Journal of Advanced Transportation, vol. 49, no. 2, pp. 195-209, 2015.

[7] A. Hegyi, B. DeSchutter, and J. Hellendoorn, "Optimal coordination of variable speed limits to suppress shock waves," IEEE Transactions on Intelligent Transportation Systems, vol. 6, no. 1, pp. 102-112, 2005.

[8] D. Li and P. Ranjitkar, "A fuzzy logic-based variable speed limit controller," Journal of Advanced Transportation, vol. 49, no. 8, pp. 913-927, 2015.

[9] D. Li, P. Ranjitkar, and Y. Zhao, "Mitigating recurrent congestion via particle swarm optimization variable speed limit controllers," KSCE Journal of Civil Engineering, vol. 23, no. 7, pp. 3174-3179, 2019.

[10] X. Yang, Y. Lin, Y. Lu, and N. Zou, "Optimal variable speed limit control for real-time freeway congestions," ProcediaSocial and Behavioral Sciences, vol. 96, pp. 2362-2372, 2013.

[11] A. Kotsialos, M. Papageorgiou, C. Diakaki, Y. Pavlis, and F. Middelham, "Traffic flow modeling of large-scale motorway networks using the macroscopic modeling tool METANET," IEEE Transactions on Intelligent Transportation Systems, vol. 3, no. 4, pp. 282-292, 2002.

[12] Y. Bie, M. Seraj, C. Zhang et al., "Improving traffic state prediction model for variable speed limit control by introducing stochastic supply and demand," Journal of Advanced Transportation, vol. 2018, Article ID 7959815, 12 pages, 2018.

[13] X. Wang, M. Seraj, Y. Bie et al., "Implementation of variable speed limits: preliminary test on whitemud drive, edmonton, Canada," Journal of Transportation Engineering, vol. 142, no. 12, Article ID 05016007, 2016.

[14] M. Yu and W. D. Fan, "Optimal variable speed limit control in connected autonomous vehicle environment for relieving freeway congestion," Journal of Transportation Engineering, Part A: Systems, vol. 145, no. 4, Article ID 04019007, 2019.

[15] M. Hadiuzzaman and T. Z. Qiu, "Cell transmission model based variable speed limit control for freeways," Canadian Journal of Civil Engineering, vol. 40, no. 1, pp. 46-56, 2013.

[16] Y. S. Jiang, B. Zhao, M. Liu et al., "A two-level model for traffic signal timing and trajectories planning of multiple CAVs in a random environment," Journal of Advanced Transportation, vol. 2021, Article ID 9945398, 13 pages, 2021.

[17] Z. H. Yao, T. Xu, Y. S. Jiang et al., "Linear stability analysis of heterogeneous traffic flow considering degradations of connected automated vehicles and reaction time," Physica A:
Statistical Mechanics and Its Applications, vol. 561, Article ID 125218, 2021.

[18] B. Alonso, Á. Ibeas, G. Musolino, C. Rindone, and A. Vitetta, "Effects of traffic control regulation on Network Macroscopic Fundamental Diagram: a statistical analysis of real data," Transportation Research Part A: Policy and Practice, vol. 126, pp. 136-151, 2019.

[19] M. Tashiro, H. Motoyama, Y. Ichioka, T. Miwa, and T. Morikawa, "Simulation analysis on optimal merging control of connected vehicles for minimizing travel time," International Journal of Intelligent Transportation Systems Research, vol. 18, no. 1, pp. 65-76, 2020.

[20] M. Wang, W. Daamen, S. P. Hoogendoorn, and B. van Arem, "Connected variable speed limits control and car-following control with vehicle-infrastructure communication to resolve stop-and-go waves," Journal of Intelligent Transportation Systems, vol. 20, no. 6, pp. 559-572, 2016.

[21] L. Pu, X. Xu, H. He, H. Zhou, Z. Qiu, and Y. Hu, "A flexible control study of variable speed limit in connected vehicle systems," International Journal of Embedded Systems, vol. 7, no. 2, pp. 180-188, 2015.

[22] Z. H. Yao, H. R. Jiang, and Y. Cheng, "Integrated schedule and trajectory optimization for connected automated vehicles in a conflict zone," IEEE Transactions on Intelligent Transportation Systems, vol. 99, 2020.

[23] H. Su, "Research about the construction of intelligent transportation system under the big data of vehicle networking," IOP Conference Series: Materials Science and Engineering, vol. 688, no. 2, Article ID 022011, 2019.

[24] Y. Zou and X. Qu, "On the impact of connected automated vehicles in freeway work zones: a cooperative cellular automaton model-based approach," Journal of Intelligent and Connected Vehicles, vol. 1, no. 6, 2018.

[25] H. T. Zhao, L. Lin, C. P. Xu et al., "Cellular automata model under Kerner's framework of three-phase traffic theory considering the effect of forward-backward vehicles in internet of vehicles," Physica A: Statistical Mechanics and its Applications, vol. 553, Article ID 124213, 2020.

[26] E. F. Grumert and A. Tapani, "Using connected vehicles in a variable speed limit system," Transportation Research Procedia, vol. 27, pp. 85-92, 2017.

[27] C. Guo, D. Li, G. Zhang, and M. Zhai, "Real-time path planning in urban area via vanet-assisted traffic information sharing," IEEE Transactions on Vehicular Technology, vol. 67, no. 7, pp. 5635-5649, 2018.

[28] M. L. Song, R. R. Li, Y. N. Wei et al., "Dynamic vehicle route guidance model under internet of vehicles," Advances in Transportation Studies, vol. 1, pp. 39-48, 2019.

[29] İ. G. Erdağı, M. A. Silgu, and H. B. Çelikoğlu, "Emission effects of cooperative adaptive cruise control: a simulation case using SUMO," EPiC Series in Computing, vol. 62, pp. 92-100, 2019.

[30] Z. Yao, R. Hu, Y. Wang et al., "Stability analysis and the fundamental diagram for mixed connected automated and human-driven vehicles," Physica A: Statistical Mechanics and Its Applications, vol. 533, 2019.

[31] X. Y. Lu, T. Z. Qiu, R. Horowitz et al., "METANET model improvement for traffic control," in Proceedings of 2011 14th International IEEE Conference on Intelligent Transportation Systems (ITSC), pp. 2148-2153, Washington, DC, USA, October 2011.

[32] M. Yu and W. Fan, "Optimal variable speed limit control at a lane drop bottleneck: genetic algorithm approach," Journal of Computing in Civil Engineering, vol. 32, no. 6, Article ID 04018049, 2018. 
[33] B. Khondaker and L. Kattan, "Variable speed limit: a microscopic analysis in a connected vehicle environment," Transportation Research Part C: Emerging Technologies, vol. 58, pp. 146-159, 2015.

[34] D. Wang, Y. Zhou, and X. He, "Radial basis function neural network-based model predictive control for freeway traffic systems," International Journal of Intelligent Systems Technologies and Applications, vol. 2, no. 4, pp. 370-388, 2007.

[35] C. Portilla, F. Valencia, J. Espinosa, A. Núñez, and B. De Schutter, "Non-linear model predictive control based on game theory for traffic control on highways," IFAC Proceedings Volumes, vol. 45, no. 17, pp. 436-441, 2012.

[36] M. Treiber, A. Hennecke, and D. Helbing, "Congested traffic states in empirical observations and microscopic simulations," Physical Review E, vol. 62, no. 2, pp. 1805-1824, 2000.

[37] M. J. Cassidy and J. Rudjanakanoknad, "Increasing the capacity of an isolated merge by metering its on-ramp," Transportation Research Part B: Methodological, vol. 39, no. 10, pp. 896-913, 2005. 